PROCEEDINGS OF THE AMERICAN MATHEMATICAL SOCIETY

Volume 125, Number 5, May 1997, Pages 1425-1434

S 0002-9939(97)03852-5

\title{
ESSENTIAL SPECTRA THROUGH LOCAL SPECTRAL THEORY
}

\author{
K. B. LAURSEN
}

(Communicated by Palle E. T. Jorgensen)

\begin{abstract}
Based on a nice observation of Eschmeier, this is a study of the use of local spectral theory in investigations of the semi-Fredholm spectrum of a continuous linear operator. We also examine the retention of the semiFredholm spectrum under weak intertwining relations; it is shown, inter alias, that if two decomposable operators are intertwined asymptotically by a quasiaffinity then they have identical semi-Fredholm spectra. The results are applied to multipliers on commutative semisimple Banach algebras.
\end{abstract}

\section{INTRODUCTION}

In [14], proof of Theorem 1.1, it was shown that the boundary of any local spectrum would necessarily belong to a particular distinguished subset of the spectrum, the Kato spectrum, although [14] did not pay much attention to this aspect, and tended to play down its use. But Jörg Eschmeier has noticed its possibilities, and pointed out how it has a bearing on the semi-Fredholm spectrum: he has observed, in a private communication to M. Neumann, cited in Neumann's excellent survey [20] as Theorem 8, that a consequence of the above containment is that every nonisolated point of the boundary $\partial \sigma_{T}(x)$ is in the semi-Fredholm spectrum $\sigma_{s F}(T)$. In this note we shall make some further observations on this. I would like to thank Ernst Albrecht and Mostafa Mbekhta for helpful comments.

\section{General LOCAL AND GLOBAL SPECTRAL THEORY}

Throughout, $X$ and $Y$ shall denote Banach spaces, and $L(X), L(Y), L(X, Y)$ the spaces of continuous linear operators on $X$, and on $Y$, respectively from $X$ to $Y$. For an operator $T \in L(X)$ recall that $T$ is called semi-Fredholm if the range $T X$ is closed and either ker $T$ or $X / T X$ is finite dimensional (and Fredholm if both are). The spectrum will be denoted by $\sigma(T)$. We shall utilize the following distinguished subsets of $\sigma(T)$ : the surjectivity spectrum $\sigma_{s u}(T):=\{\lambda \in \mathbf{C}:(T-\lambda) X \neq X\}$, the semi-Fredholm spectrum $\sigma_{s F}(T):=\{\lambda \in \mathbf{C}: T-\lambda$ is not semi-Fredholm on $X\}$, and the (Fredholm) essential spectrum $\sigma_{e}(T):=\{\lambda \in \mathbf{C}: T-\lambda$ is not Fredholm on $X\}$. Obviously $\sigma_{s F}(T) \subseteq \sigma_{e}(T)$. We shall also make use of the Kato resolvent $\rho_{K}(T):=\left\{\lambda \in \mathbf{C}:(T-\lambda) X\right.$ is closed and $\left.\operatorname{ker}(T-\lambda) \subseteq \bigcap_{n=1}^{\infty}(T-\lambda)^{n} X\right\}$ and its

Received by the editors June 28, 1995 and, in revised form, November 17, 1995.

1991 Mathematics Subject Classification. Primary 47A10, 47A11, 47B40; Secondary 43A22, 46J10, 47A53. 
complement, the Kato spectrum $\sigma_{K}(T):=\mathbf{C} \backslash \rho_{K}(T)$. This is all explained in e.g. [16]. The following local concepts may be found, for instance in [13], [14].

Local spectral theory is based on the existence of analytic solutions $f: U \curvearrowright X$ to the equation $(T-\lambda) f(\lambda)=x$ on open subsets $U \subseteq \mathbf{C}$, for a given operator $T \in L(X)$ and a given element $x \in X$. We use this in defining the global spectral subspaces: for a closed set $F \subseteq \mathbf{C}$ let $\mathcal{X}_{T}(F):=\{x \in X:(T-\lambda) f(\lambda)=x$ has an analytic solution $f: \mathbf{C} \backslash F \curvearrowright X\}$, and we use it in defining the local spectrum $\sigma_{T}(x):=\mathbf{C} \backslash \bigcup\{U:(T-\lambda) f(\lambda)=x$ has an analytic solution $f: U \curvearrowright X$ on the open subset $U \subseteq \mathbf{C}\}$.

If for every $x \in X$ any two such solutions always coincide on the intersection of their domains of definition, then $T$ is said to have the single valued extension property SVEP. If all the spaces $\mathcal{X}_{T}(F)$ are closed, whenever $F \subseteq \mathbf{C}$ is closed, then we say that $T$ has property $(C)$. Property $(C)$ entails SVEP [13, Proposition 1.2]. If $T$ satisfies the condition that for every pair of open subsets $U, V \subseteq \mathbf{C}$ for which $\mathbf{C}=U \cup V$ the space $X$ may be written as $X=\mathcal{X}_{T}(\bar{U})+\mathcal{X}_{T}(\bar{V})$ then $T$ is said to have property $(\delta)$. The concept of decomposability of the operator $T$ may be introduced in a variety of ways; here it will be expeditious to say that $T$ is decomposable if $T$ has both properties $(C)$ and $(\delta)$. Prominent examples of decomposable operators are normal operators on Hilbert space, and compact operators on any Banach space. Indeed all operators with totally disconnected spectrum are decomposable, hence in particular so are quasi-nilpotent and algebraic operators.

The Albrecht-Eschmeier duality theory, [3], says that an operator has property $(\delta)$ precisely when its (pre-)adjoint operator has a property called $(\beta)$. For an operator $T \in L(X)$ possession of property $(\beta)$ means that any sequence of analytic functions $f_{n}: U \curvearrowright X$, where $U \subseteq \mathbf{C}$ is an open set, for which $(T-\lambda) f_{n}(\lambda) \rightarrow 0$, uniformly on every compact subset of $U$, itself converges to 0 , in that same topology. Property $(\beta)$ entails property $(C)$, [13, Proposition 1.2], and is entailed by decomposability. Thus, decomposability is also the same as properties $(\beta)$ and $(\delta)$ together. These concepts are even more intimately related: another significant feature of [3] is the characterization of the class of operators with property $(\beta)$ as identical to the class of restrictions to invariant subspaces of decomposable operators. This also means the characterization of the class of operators with property $(\delta)$ as the same as all quotients (modulo invariant subspaces, of course) of decomposable operators.

Our starting point is the following slight generalization of Eschmeier's result.

Theorem 1. If $X$ is a Banach space, if $T \in L(X)$ and if $\lambda \in \mathbf{C}$ is a limit point of a sequence of distinct points $\lambda_{n} \in \partial \sigma_{T}\left(x_{n}\right)$ where $x_{n} \in X$, then $\lambda$ is in the semi-Fredholm spectrum $\sigma_{s F}(T)$.

Proof. This is an adaptation of Eschmeier's proof, which we are grateful to M. Neumann for telling us about, and to J. Eschmeier for permitting us the use of. Let $\lambda \in \mathbf{C}$ satisfy the stated assumptions. Clearly, we have that $\lambda \in \sigma(T)$, so if $\lambda \notin \sigma_{s F}(T)$, then $\lambda$ belongs to a connected component $\Omega$ of the semi-Fredholm region of $T$, and we may consider $\Omega$ to be a subset of $\sigma(T)$. Also we may assume that $\lambda_{n} \in \Omega$ for all $n \in \mathbf{N}$. The set $\mathcal{S}(\Omega)$ of points of discontinuity of the function $z \curvearrowright \operatorname{dim} \operatorname{ker}(T-z), z \in \Omega$, is a discrete subset of $\Omega$, in fact, by [21, p.

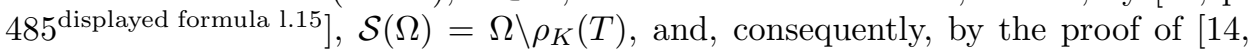
Theorem 1.1], $\lambda_{n} \in \mathcal{S}(\Omega)$ for all $n \in \mathbf{N}$. Since $\mathcal{S}(\Omega)$ is discrete, this implies that $\lambda \notin \Omega$, a contradiction. Consequently, $\lambda \in \sigma_{s F}(T)$. 
Thus, if $\lambda \in \sigma(T) \backslash \sigma_{s F}(T)$ then for every $x \in X$ for which $\lambda \in \sigma_{T}(x)$ we see that either $\lambda$ is isolated in $\sigma_{T}(x)$, or $\lambda$ is an interior point of $\sigma_{T}(x)$. We shall examine the points outside the semi-Fredholm spectrum in more detail.

Theorem 2. Let $T \in L(X)$. If $\lambda \notin \sigma_{s F}(T)$ then $\mathcal{X}_{T}(\{\lambda\})$ is closed if and only if $\mathcal{X}_{T}(\{\lambda\})$ is finite dimensional. In particular, if $T$ has property $(C)$ and $\lambda \notin \sigma_{s F}(T)$, then $\mathcal{X}_{T}(\{\lambda\})$ is finite dimensional. Moreover, in this event, $\mathcal{X}_{T}(\{\lambda\}) \neq\{0\}$ if and only if $\lambda$ is an eigenvalue of $T$.

Proof. For the first part the one direction is trivial, so we may suppose that $\lambda \in$ $\sigma(T) \backslash \sigma_{s F}(T)$ and that $\mathcal{X}_{T}(\{\lambda\})$ is closed. We observe that $\sigma\left(T \mid \mathcal{X}_{T}(\{\lambda\})\right)=\{\lambda\}$, i.e. that $(T-\lambda) \mid \mathcal{X}_{T}(\{\lambda\})$ is quasi-nilpotent: this follows, for instance, from the fact that $\mathcal{X}_{T}(\{\lambda\})=\left\{x \in X:\left\|(T-\lambda)^{n} x\right\|^{1 / n} \rightarrow 0\right.$ as $\left.n \rightarrow \infty\right\}$ [19]. If $\mu \neq \lambda$ then $\mu$ is not an eigenvalue of $T \mid \mathcal{X}_{T}(\{\lambda\})$, because $T x=\mu x$ implies that $(T-\lambda) x=(\mu-\lambda) x$, and hence $\left\|(T-\lambda)^{n} x\right\|^{1 / n}=\left\|(\mu-\lambda)^{n} x\right\|^{1 / n}=|\mu-\lambda|\|x\|^{1 / n} \rightarrow 0$, if $x \in \mathcal{X}_{T}(\{\lambda\})$, and this means that $x=0$. Since $(T-\mu) \mid \mathcal{X}_{T}(\{\lambda\})$ is surjective when $\lambda \neq \mu$, by analytic uniqueness (if $(T-\mu) f(\mu)=x$ for all $\mu \neq \lambda$, where $f$ is an analytic function, then $f(\mu)=(T-\mu)^{-1} x \in \mathcal{X}_{T}(\{\lambda\})$ for all $\mu$ of sufficiently large absolute value, hence $f(\mu) \in \mathcal{X}_{T}(\{\lambda\})$, because $\mathcal{X}_{T}(\{\lambda\})$ is assumed closed), the quasi-nilpotence of $(T-\lambda) \mid \mathcal{X}_{T}(\{\lambda\})$ follows. Since $(T-\lambda) \mid \mathcal{X}_{T}(\{\lambda\})$ is Fredholm, it follows that $(T-\lambda) \mid \mathcal{X}_{T}(\{\lambda\})$ is nilpotent $\left[23\right.$, Proposition 2.5], hence that $\mathcal{X}_{T}(\{\lambda\})$ is finite dimensional.

If $T$ has property $(C)$ then, for any $\lambda \in \mathbf{C}$, the spectral subspace $\mathcal{X}_{T}(\{\lambda\})$ is closed and therefore, by the first part of this proof, finite dimensional, if $\lambda \notin \sigma_{s F}(T)$.

For the last claim, it is clear that if $\lambda \in \sigma_{p}(T)$ then $\operatorname{ker}(T-\lambda)$, and hence the larger space $\mathcal{X}_{T}(\{\lambda\})$, is non-zero. Conversely, if $\mathcal{X}_{T}(\{\lambda\}) \neq\{0\}$ then $\sigma\left(T \mid \mathcal{X}_{T}(\{\lambda\})\right)$ $=\{\lambda\}$ forces $\lambda$ to be an eigenvalue of $T$.

Thus, finite dimensionality of $\mathcal{X}_{T}(\{\lambda\})$ is necessary for $\lambda \notin \sigma_{s F}(T)$, for a rather large class of operators, those with property $(C)$; but it is not sufficient, for this class: for non-isolated points of the spectrum it is not true that finite dimensionality of $\mathcal{X}_{T}(\{\lambda\})$ implies that $\lambda \notin \sigma_{s F}(T)$, as the example of the right shift $R$ on $\ell^{2}(\mathbf{N})$ shows: $\sigma_{s F}(R)=\mathbf{T}$, but $\ell^{2}(\mathbf{N})_{R}(\{\lambda\})=\{0\}$ for every $\lambda \in \mathbf{C}$, [11, Proposition 2].

If $\lambda$ is an isolated point of the spectrum, however, finite dimensionality of $\mathcal{X}_{T}(\{\lambda\})$ is a characterization of non-membership of the semi-Fredholm spectrum, as well as of the essential spectrum. To record this properly we need some terminology. We shall consider the space $\mathcal{K}_{T}(\{\lambda\}):=\left\{x \in X: \lambda \in \mathbf{C} \backslash \sigma_{T}(x)\right\}$. It is straightforward that this is a linear space; in fact, if the open disc of radius $r$, centered at $\lambda \in \mathbf{C}$ is denoted by $D(\lambda, r)$, then it is clear that $\mathcal{K}_{T}(\{\lambda\})=\bigcup_{n=1}^{\infty} \mathcal{X}_{T}\left(\mathbf{C} \backslash D\left(\lambda, \frac{1}{n}\right)\right)$. This space was introduced, differently, by Mbekhta, in [16], and originally (for $\lambda=0$ ) denoted by $K(T)$. In [16] it is also pointed out that $(T-\lambda) \mathcal{K}_{T}(\{\lambda\})=\mathcal{K}_{T}(\{\lambda\})$. We then have the following.

Lemma 1. Let $T \in L(X)$. A point $\lambda \in \mathbf{C}$ is isolated in the spectrum $\sigma(T)$ if and only if $\mathcal{K}_{T}(\{\lambda\})$ is closed and $X=\mathcal{X}_{T}(\{\lambda\}) \oplus \mathcal{K}_{T}(\{\lambda\})$. Consequently, if $\lambda \in \sigma(T)$ is isolated then $\lambda \notin \sigma_{s F}(T)$ if and only if $\lambda \notin \sigma_{e}(T)$ if and only if $\mathcal{X}_{T}(\{\lambda\})$ is finite dimensional.

Proof. The first part is [22, Theorem 4], with the notational changes indicated above. For the second part suppose that $\mathcal{K}_{T}(\{\lambda\})$ is closed and $X=\mathcal{X}_{T}(\{\lambda\}) \oplus$ $\mathcal{K}_{T}(\{\lambda\})$. We note that it is always the case that $\operatorname{ker}(T-\lambda) \subseteq \mathcal{X}_{T}(\{\lambda\})$, cf. e.g. 
[14, Proposition 1.3 (a)]. Moreover, as just mentioned, $(T-\lambda) \mathcal{K}_{T}(\{\lambda\})=\mathcal{K}_{T}(\{\lambda\})$. This makes it clear that if $\mathcal{X}_{T}(\{\lambda\})$ is finite dimensional then $\lambda \notin \sigma_{e}(T)$, and hence $\lambda \notin \sigma_{s F}(T)$. For the converse note that when $X=\mathcal{X}_{T}(\{\lambda\}) \oplus \mathcal{K}_{T}(\{\lambda\})$ and $\mathcal{K}_{T}(\{\lambda\})$ is closed, then so is $\mathcal{X}_{T}(\{\lambda\})$. Consequently, if $\lambda \notin \sigma_{s F}(T)$ then $\mathcal{X}_{T}(\{\lambda\})$ is finite dimensional, by Theorem 2 , and $\lambda \notin \sigma_{e}(T)$.

Theorem 3. If $T \in L(X)$ is an operator with property $(\delta)$, then all cluster points of $\sigma_{s u}(T)$ are in the semi-Fredholm spectrum $\sigma_{s F}(T)$.

Proof. If $\lambda_{0}$ is a cluster point of $\sigma_{s u}(T)$, then the set $\overline{D\left(\lambda_{0}, r\right)} \cap \sigma_{s u}(T)$ is infinite, for any positive radius $r$. We fix arbitrarily such a radius $r$. Since $\sigma_{s F}(T)$ is closed it suffices to show that $\overline{D\left(\lambda_{0}, r\right)}$ contains points of $\sigma_{s F}(T)$. By Eschmeier's observation (leading to Theorem 1), it will suffice to produce an element $x \in X$ with infinite local spectrum $\sigma_{T}(x) \subseteq \overline{D\left(\lambda_{0}, r\right)}$. Choose two closed concentric discs $\overline{D\left(\lambda_{0}, r_{1}\right)}, \overline{D\left(\lambda_{0}, r_{2}\right)}$ with positive radii satisfying $0<r_{1}<r_{2}<r$. Also choose an element $z \in X$ such that $\sigma_{T}(z)=\sigma_{s u}(T)$. This is possible, by [15, Lemma 2]. Since $T$ has property $(\delta), X=\mathcal{X}_{T}\left(\overline{D\left(\lambda_{0}, r\right)}\right)+\mathcal{X}_{T}\left(\mathbf{C} \backslash D\left(\lambda_{0}, r_{2}\right)\right)$ and consequently there are elements $x$ and $y$ in $X$ which satisfy that $z=x+y$ and that $\sigma_{T}(x) \subseteq \overline{D\left(\lambda_{0}, r\right)}$, while $\sigma_{T}(y) \cap \overline{D\left(\lambda_{0}, r_{1}\right)}=\emptyset$. Clearly, $\sigma_{T}(z) \subseteq \sigma_{T}(x) \cup \sigma_{T}(y)$, and hence $\sigma_{s u}(T) \cap$ $\overline{D\left(\lambda_{0}, r_{1}\right)}=\sigma_{T}(z) \cap \overline{D\left(\lambda_{0}, r_{1}\right)} \subseteq \sigma_{T}(x) \cap \overline{D\left(\lambda_{0}, r_{1}\right)}$, because $\sigma_{T}(y) \cap \overline{D\left(\lambda_{0}, r_{1}\right)}=\emptyset$. This shows that $\sigma_{T}(x) \cap \overline{D\left(\lambda_{0}, r_{1}\right)}$, hence also $\sigma_{T}(x) \cap \overline{D\left(\lambda_{0}, r\right)}=\sigma_{T}(x)$, is an infinite set. Thus $D$ contains a cluster point of $\partial \sigma_{T}(x)$, i.e. a point of $\sigma_{s F}(T)$.

Corollary 1. If $T \in L(X)$ is an operator that has both SVEP and property $(\delta)$, in particular if $T$ is a decomposable operator, then $\sigma_{s F}(T)=\sigma_{e}(T)$, and consists of all cluster points of $\sigma(T)$ along with the isolated points $\lambda \in \sigma(T)$ for which $\mathcal{X}_{T}(\{\lambda\})$ is infinite dimensional.

Proof. Since $T$ has SVEP it follows that $\sigma(T)=\sigma_{s u}(T)$ [15, Corollary of Lemma $3]$, and the conclusion is then immediate from Theorem 3 and Lemma 1.

Obviously, this corollary holds for any operator $T$ with property $(\delta)$ for which $\sigma(T)=\sigma_{s u}(T)$. However, without this equality the conclusion of Corollary 1 is not true. The right shift $R$, mentioned a little while ago, is a step towards an example: the set of cluster points of the spectrum equals the closed unit disc $\mathbf{D}$, while $\sigma_{s F}(R)=\mathbf{T}$. Since $R$ is an isometry, it has property $(\beta)$ [9], and consequently its adjoint, the left shift $L$, has property $(\delta)$, by the Albrecht-Eschmeier duality theory [3]. An operator and its adjoint have identical spectra and identical semiFredholm spectra, and hence $\sigma_{s F}(L)=\mathbf{T} \neq \mathbf{D}$.

Remark 1. Better results than Corollary 1 have been known for some time. Based either on a technique employed in [4, proof of Proposition 4.6], or on work described in [17, Corollary 1.8], we have that if $T \in L(X)$ and its adjoint $T^{*} \in L\left(X^{*}\right)$ both have SVEP then every point of $\sigma(T) \backslash \sigma_{s F}(T)$ is an isolated point. Thus $\sigma_{s F}(T)$ consists of all cluster points of $\sigma(T)$ along with the isolated points $\lambda \in \sigma(T)$ for which $\mathcal{X}_{T}(\{\lambda\})$ is infinite dimensional. With SVEP only for $T$ this conclusion is not true. The right shift $R$ is an example. This more general version of Corollary 1 is not particularly useful in the study of intertwining relations, because no spectral preservation results are known yet for operators which along with their adjoints have SVEP. 


\section{INTERTWINING}

Similarity of operators $S \in L(Y)$ and $T \in L(X)$ is a significant equivalence relation of operator theory and one of its many accompanying facts is the preservation of spectra. If similarity is relaxed to intertwining, i.e. to the existence of an operator $A \in L(X, Y)$, an intertwiner, such that $S A=A T$, then spectral preservation is no longer guaranteed although, by a classical result, cf. e.g. [13, p. 488], the spectra of $S$ and $T$ must overlap. For essential spectra the best known general result is about quasi-similar operators. The operators $S$ and $T$ are said to be quasisimilar if there are quasi-affinities $A \in L(X, Y)$, respectively $B \in L(Y, X)$, which intertwine, i.e. for which $S A=A T$ and $T B=B S$ (a quasi-affinity is a linear map which is injective and has norm-dense range). In 1988 Herrero showed, in [7], that no component of either of the essential spectra of two quasi-similar operators can avoid touching the other essential spectrum. How this result fares if we relax the intertwining to that of asymptotic intertwining (to be introduced now), and/or consider instead the semi-Fredholm spectrum, is not yet known. Here we shall deal with asymptotic intertwining, but restrict our attention to intertwined operators with appropriate spectral decomposition properties. First recall that operators $S \in L(Y)$ and $T \in L(X)$ are said to be intertwined asymptotically by $A \in L(X, Y)$, if the commutator $C(S, T) \in L(L(X, Y))$, defined by $C(S, T)(A):=S A-A T$, satisfies the condition that $\left\|C(S, T)^{n}(A)\right\|^{1 / n} \rightarrow 0$ as $n \rightarrow \infty$. Any $A$ for which $C(S, T)^{n}(A)=0$ for some $n \in \mathbf{N}$, e.g. an intertwiner, will be an example, and to exemplify this non-trivially, note that any quasi-nilpotent operator and the zero operator are intertwined asymptotically by the identity operator. Asymptotic intertwining is the same as saying that $A$ is an element of the glocal spectral subspace $\mathcal{L}(\mathcal{X}, \mathcal{Y})_{C(S, T)}(\{0\})$, by the description of these spaces [19], also referred to in the proof of Theorem 2.

A fact about asymptotic intertwining, which dates back to [5] and which we shall use repeatedly, and often tacitly, is that if $S$ and $T$ are intertwined asymptotically by $A$, then $A \mathcal{X}_{T}(F) \subseteq \mathcal{Y}_{S}(F)$ for any closed set $F \subseteq \mathbf{C}$; in the present context this was noted in [13, Proposition 2.2].

If $S$ and $T$ are intertwined asymptotically by a quasi-affinity $A$, while $T$ and $S$ are intertwined asymptotically by a quasi-affinity $B \in L(Y, X)$, then we say that $S$ and $T$ are asymptotically quasi-similar.

Theorem 4. If $S \in L(Y)$ and $T \in L(X)$ are intertwined asymptotically by a quasiaffinity $A$ and if $K:=\sigma(T)=\sigma(S)$ then an isolated point $\lambda \in K$ is in $\sigma_{s F}(T)$ if and only if $\lambda$ is in $\sigma_{s F}(S)$, and this occurs if and only if $\lambda$ is in $\sigma_{e}(T)$, hence if and only if $\lambda$ is in $\sigma_{e}(S)$.

Proof. By Lemma 1 we have to show that if $\lambda \in K$ is an isolated point then $\mathcal{X}_{T}(\{\lambda\})$ is finite dimensional if and only if this is the case for $\mathcal{Y}_{S}(\{\lambda\})$. If $\mathcal{Y}_{S}(\{\lambda\})$ is finite dimensional, this is clear because $A \mathcal{X}_{T}(\{\lambda\}) \subseteq \mathcal{Y}_{S}(\{\lambda\})$, and $A$ is injective. On the other hand, if $\mathcal{X}_{T}(\{\lambda\})$ is finite dimensional, then so is $A \mathcal{X}_{T}(\{\lambda\}) \subseteq \mathcal{Y}_{S}(\{\lambda\})$. Making use of the density assumption on $A$, it is then elementary to see that in fact $A \mathcal{X}_{T}(\{\lambda\})=\mathcal{Y}_{S}(\{\lambda\})$.

We now come to the main results of this section. They are, in part, generalizations of $[18]$. 
Theorem 5. If $S \in L(Y)$ and $T \in L(X)$ have $S V E P$ and property $(\delta)$, and if $S$ and $T$ are asymptotically intertwined by an injective map $A$, then $\sigma(T) \subseteq \sigma(S)$ and $\sigma_{s F}(T)=\sigma_{e}(T) \subseteq \sigma_{s F}(S)=\sigma_{e}(S)$.

Proof. We note first that $\sigma(T) \subseteq \sigma(S)$, by [13, Theorem 4.1 (c)]. Any cluster point of the set $\sigma(T)$ will be in the semi-Fredholm spectrum of both operators, by Corollary 1, and an isolated point $\lambda$ of $\sigma(T)$ will be either non-isolated in $\sigma(S)$ (in which case it belongs to $\left.\sigma_{s F}(S)\right)$, or it will be isolated in $\sigma(S)$ as well. If, in this latter case $\lambda \notin \sigma_{s F}(S)$ then $\mathcal{Y}_{S}(\{\lambda\})$ is finite dimensional, and since $A \mathcal{X}_{T}(\{\lambda\}) \subseteq$ $\mathcal{Y}_{S}(\{\lambda\})$ and $A$ is injective, $\mathcal{X}_{T}(\{\lambda\})$ is also finite dimensional. Thus $\lambda \notin \sigma_{s F}(T)$, by Lemma 1.

Corollary 2. If $S \in L(Y)$ and $T \in L(X)$ have $S V E P$ and property ( $\delta$ ), and if $S$ and $T$ are asymptotically quasi-similar, then $\sigma(T)=\sigma(S)$ and $\sigma_{s F}(T)=\sigma_{e}(T)=$ $\sigma_{s F}(S)=\sigma_{e}(S)$.

Proof. The equality $\sigma(T)=\sigma(S)$ is [13, Corollary 4.5], or two applications of Theorem 5. Similarly, $\sigma_{s F}(T)=\sigma_{e}(T)=\sigma_{s F}(S)=\sigma_{e}(S)$ is immediate from Theorem 5 .

A straightforward application of the Albrecht-Eschmeier duality, combined with the fact that two operators are intertwined asymptotically by an operator if and only if their adjoints are intertwined asymptotically by the adjoint of the intertwining map, yields the following result.

Corollary 3. If $S \in L(Y)$ and $T \in L(X)$ have property $(\beta)$ and their adjoint operators have $S V E P$, and if $S$ and $T$ are asymptotically quasi-similar, then $\sigma(T)=$ $\sigma(S)$ and $\sigma_{s F}(T)=\sigma_{e}(T)=\sigma_{s F}(S)=\sigma_{e}(S)$.

Proof. As mentioned, if $S$ and $T$ are intertwined asymptotically by $A$ then $T^{*}$ and $S^{*}$ are intertwined asymptotically by $A^{*}$. Since $T^{*}$ and $S^{*}$ have property $(\delta)$ and SVEP, and since spectra, essential, and semi-Fredholm spectra are preserved under the passage between adjoints and pre-adjoints, two applications of Theorem 5 will complete the argument.

The next result, Theorem 6 , also concludes equality of spectra and semi-Fredholm spectra, but the two operators do not appear symmetrically in the assumptions, and we can relax the condition on the intertwining map to that of quasi-affinity. Note also that there is no explicit assumption of SVEP on $T$.

Theorem 6. If $S \in L(Y)$ and $T \in L(X)$ are asymptotically intertwined by a quasiaffinity $A$, and if $S$ has property $(C)$ while $T$ has property $(\delta)$, then $\sigma(T)=\sigma(S)$ and $\sigma_{s F}(T)=\sigma_{e}(T)=\sigma_{e}(S)=\sigma_{s F}(S)$. The latter set consists of the cluster points of the former and, additionally, those isolated points $\lambda$ for which the spectral subspace $\mathcal{X}_{T}(\{\lambda\})$ (and/or $\left.\mathcal{Y}_{S}(\{\lambda\})\right)$ is infinite dimensional.

Proof. The equality of the spectra is Corollary 4.2 of [13]. Moreover, by [13, Theorem $4.1(\mathrm{a})], K:=\sigma(T)=\sigma_{s u}(T)$, and since $S$ has SVEP we also have $\sigma(S)=\sigma_{s u}(S)=K$. Thus, from Theorem 3 we conclude that all cluster points of $K$ are in $\sigma_{s F}(T)$. We claim that all cluster points of $K$ are in $\sigma_{s F}(S)$ as well. Let $\lambda$ be a cluster point of $K$, and let $D(\lambda, r)$ be an arbitrary disc centered at $\lambda$. By Theorem 1, it suffices, because $\sigma_{s F}(S)$ is a closed set, to show that $\overline{D(\lambda, r)}$ contains infinitely many distinct boundary points of local spectra. By the proof of Theorem 
3, there is an element $x \in X$ for which $\sigma_{T}(x)$ is an infinite subset of $\overline{D(\lambda, r)}$, and since $A \mathcal{X}_{T}(\overline{D(\lambda, r)}) \subseteq \mathcal{Y}_{S}(\overline{D(\lambda, r)})$, it follows that $\sigma_{S}(A x) \subseteq \overline{D(\lambda, r)}$. If $\sigma_{S}(A x)$ is infinite, we are done. Otherwise, a slight refinement of this reasoning will complete the argument: there is a sequence of distinct points of $K \cap \overline{D(\lambda, r)}$ converging to $\lambda$. Hence there is a sequence of pairwise disjoint closed discs $\overline{D\left(\lambda_{n}, r_{n}\right)}$, centered at $\lambda_{n}$. For each $n \in \mathbf{N}$ we can find $x_{n} \in X$ such that $\lambda_{n} \in \sigma_{T}\left(x_{n}\right) \subseteq \overline{D\left(\lambda_{n}, r_{n}\right)}$. Since $\emptyset \neq \sigma_{S}\left(A x_{n}\right) \subseteq \overline{D\left(\lambda_{n}, r_{n}\right)}$, Theorem 1 implies that $\lambda \in \sigma_{s F}(S)$. This establishes that all cluster points of $K$ belong to both semi-Fredholm spectra, and consequently to both essential spectra. For the isolated points we appeal to Theorem 4 .

We extract a statement about decomposable operators from Theorem 6 , rather than from Theorem 5, to retain the lack of symmetry in the assumptions on the intertwining of $S$ and $T$ : it is not necessary to suppose (asymptotic) quasi-similarity. This is analogous to the assumptions of the classical result on this area, [5, Theorem 2.4.4] (of which this Corollary is a generalization) which establishes equality of the spectra of two decomposable operators intertwined by a quasi-affinity.

Corollary 4. If $S \in L(Y)$ and $T \in L(X)$ are decomposable operators which are asymptotically intertwined by a quasi-affinity $A$, then $\sigma_{s F}(T)=\sigma_{e}(T)=\sigma_{e}(S)=$ $\sigma_{s F}(S)$, and this set consists of the cluster points of their common spectrum and those isolated points $\lambda$ for which the spectral subspace $\mathcal{X}_{T}(\{\lambda\})\left(\right.$ and/or $\left.\mathcal{Y}_{S}(\{\lambda\})\right)$ is infinite dimensional.

Remark 2. What is really being examined here is the relation between the surjectivity spectra and the semi-Fredholm spectra. Since the most satisfying conclusions emerge when the surjectivity spectrum coincides with the spectrum, these conclusions are obtained at the expense of some generality, cf. the assumption of SVEP alongside property $(\delta)$, e.g. in Corollary 1 . For several reasons, among them that other approaches, e.g. [18], avoid explicit reliance on SVEP, it would be natural to investigate further the relations between the surjectivity spectra of intertwined operators.

\section{Multipliers}

Consider a commutative Banach algebra $A$ with maximal ideal space (i.e. space of multiplicative linear functionals, equipped with the weak* topology) $\Delta(A)$. The Gelfand transform, defined on $A$ by $a \curvearrowright \widehat{a} \in C_{0}(\Delta(A))$, where $C_{0}(\Delta(A))$ is the usual algebra of continuous complex valued functions, vanishing at infinity on the locally compact Hausdorff space $\Delta(A)$, and where $\widehat{a}(\phi):=\phi(a)$ for every $a \in A$ and every $\phi \in \Delta(A)$, is a norm decreasing algebra homomorphism and an injection exactly when $A$ is semisimple. By a multiplier $T$ on $A$ we mean a continuous linear operator for which the identity $a T(b)=T(a) b$ holds for all $a, b \in A$. Obvious examples (in the present commutative situation) are the operators given by multiplication by a fixed element of $A$. The best non-trivial examples of multipliers are probably obtained from group algebras: if $G$ denotes a locally compact abelian group and $L^{1}(G)$ the group algebra of Haar-integrable complex valued functions, organized as an algebra by means of convolution multiplication, then the multipliers on $L^{1}(G)$ are exactly the convolution algebra of bounded regular complex valued measures on $G$. All this may be found in the standard reference on multipliers [8].

We shall denote the set of multipliers on $A$ by $M(A)$. Associated to a multiplier $T \in M(A)$ is a bounded continuous function $\widehat{T}$ on $\Delta(A)$, with the property that 
$\widehat{T a}=\widehat{T} \widehat{a}$ for all $a \in A$. Thus the Gelfand transform establishes an intertwining relationship between the multiplier $T$ and the multiplication operator $L_{\widehat{T}}$ defined on $C_{0}(\Delta(A))$ by $L_{\widehat{T}}(f):=\widehat{T} f$ for all $f \in C_{0}(\Delta(A))$, and it is this simple observation that makes the above theory readily applicable to multipliers.

The semisimplicity of $A$ makes it plain to see that any multiplier $T \in M(A)$ has SVEP, so $\sigma(T)=\sigma_{s u}(T)$. But $T$ does not necessarily have $(\delta)$, so $\sigma_{s F}(T)$ is not immediately identifiable by the methods of this paper. We observe that a multiplier $T$ is Fredholm if and only if it is negative semi-Fredholm, i.e. has range $T A$ of finite codimension. This follows since $T A \cap \operatorname{ker} T=\{0\}$. In fact, the semisimplicity makes it easy to see that $\operatorname{ker} T=\operatorname{ker} T^{2}$, i.e. that a multiplier $T$ has finite ascent (actually has ascent $\leq 1$ ), and from this the equality $T A \cap \operatorname{ker} T=\{0\}$ is immediate. Note that these remarks apply to multipliers $T-\lambda$, hence make it possible to identify the (Fredholm) essential spectrum with the 'negative semi-Fredholm' spectrum.

Aiena [1] has studied the Weyl spectrum $\sigma_{w}(T)$ of a multiplier $T \in M(A)$, where $\lambda \notin \sigma_{w}(T)$ if and only if $T-\lambda$ is Fredholm and has index 0 , i.e. $\operatorname{dim} \operatorname{ker}(T-\lambda)=$ $\operatorname{codim}(T-\lambda) A<\infty$. Obviously $\sigma_{s F}(T) \subseteq \sigma_{w}(T)$, and the example of the disc algebra $A(\mathbf{D})$ shows that these two spectra may well be distinct: for $(T f)(z):=$ $z f(z)$, where $f \in A(\mathbf{D})$ we have that $\sigma(T)=\mathbf{D}$, and the interior of $\mathbf{D}$ consists of points $\lambda$ for which $(T-\lambda)$ is Fredholm, but the index is -1 , hence $\sigma_{w}(T)=\mathbf{D}$ while $\sigma_{s F}(T)=\mathbf{T}$. On the other hand, with additional assumptions the two spectra do coincide; for instance, if $A$ is a regular semisimple commutative and Tauberian Banach algebra, then $\sigma_{s F}(T)=\sigma_{w}(T)$ [2, Theorem 4.5]. Moreover, with these assumptions on $A$, as well as the assumption that $\Delta(A)$ contains no isolated points then $\sigma_{s F}(T)=\sigma_{w}(T)=\sigma(T)$. The technical terms used in these last statements are defined, for instance, in [2].

For multipliers we have the following amelioration of Lemma 1.

Proposition 1. Let $T \in M(A)$, where $A$ is a commutative semisimple Banach algebra and let $\lambda \in \mathbf{C}$ be an isolated point of $\sigma(T)$. Then $\lambda \notin \sigma_{s F}(T)$ if and only if $\operatorname{dim} \operatorname{ker}(T-\lambda)<\infty$ and this happens if and only if $\{\phi \in \Delta(A): \widehat{T}(\phi)=\lambda\}$ is finite, and if and only if $\lambda \notin \sigma_{e}(T)$.

Proof. The first equivalence is a consequence of [10, Theorems 5, 6, 10]. For the second equivalence we note that if $\lambda \in \sigma(T)$ is an isolated point for which $T-\lambda$ is semi-Fredholm then, by Lemma 1 , hull $((T-\lambda) A):=\{\phi \in \Delta(A): \phi((T-\lambda) A)=$ $\{0\}\}$ is finite and this hull is easily seen to equal $\{\phi \in \Delta(A): \widehat{T}(\phi)=\lambda\}$. Conversely, if $\{\phi \in \Delta(A): \widehat{T}(\phi)=\lambda\}$ is a finite set, its points are clopen and it is immediate that this set supports $\operatorname{ker}(T-\lambda)$, so that $\operatorname{ker}(T-\lambda)$ is finite dimensional.

For multipliers on commutative semisimple Banach algebras we can improve on Theorem 6: the intertwining map is the Gelfand transform and only injectivity (not dense range) will be used. Thus we do not need to restrict attention to, say, self adjoint algebras where the Stone-Weierstrass theorem might be invoked.

Theorem 7. If $T \in M(A)$ is a multiplier with property $(\delta)$ on the commutative semisimple Banach algebra $A$ then $\sigma(T)=[\widehat{T}(\Delta(A))]^{-}$and $\sigma_{s F}(T)=\sigma_{e}(T)=$ $\sigma_{s F}\left(L_{\widehat{T}}\right)=\sigma_{e}\left(L_{\widehat{T}}\right)$. This latter set consists of all cluster points of $\widehat{T}(\Delta(A))$ together with all isolated points $\lambda$ of $\widehat{T}(\Delta(A))$ for which $\{\phi \in \Delta(A): \widehat{T}(\phi)=\lambda\}$ is an infinite set. 
Proof. The fact that $\sigma(T)=[\widehat{T}(\Delta(A))]^{-}$is proved in [12, Proposition 3]. For the rest of the argument we can quote the proof of Theorem 6 verbatim, except that the description of the isolated points in the spectrum of course comes from Proposition 1.

Without an assumption such as $(\delta)$, it is not true that $\sigma_{s F}(T)=\sigma_{s F}\left(L_{\widehat{T}}\right)$, as the example of multiplication by the independent variable on the disc algebra shows. Here $\sigma_{s F}(T)$ is the unit circle while $\sigma_{s F}\left(L_{\widehat{T}}\right)$ is the whole unit disc.

The property of a multiplier that $\sigma(T)=[\widehat{T}(\Delta(A))]^{-}$is called natural spectrum and has received a fair amount of attention, e.g. [6], [12], [24]. The references [6] and [12] are also good sources of information on circumstances under which a multiplier has property $(\delta)$.

\section{REFERENCES}

[1] P Aiena: Riesz multipliers on commutative semisimple Banach algebras, Arch.Math. 54 (1990), 293-303. MR 91e:46064

[2] P Aiena, K B Laursen: Multipliers with closed range on regular commutative Banach algebras, Proc.Amer. Math.Soc. 121 (1994), 1039-1048. MR 94j:46051

[3] E Albrecht, J Eschmeier: Analytic functional models and local spectral theory (manuscript, 1987)

[4] E Albrecht, R D Mehta: Some remarks on local spectral theory, J. Operator Theory, 12 (1984), no. 2, 285-317. MR 85m:47031

[5] I Colojoara, C Foias: Theory of generalized spectral operators, Gordon and Breach, New York 1968. MR 52:15085

[6] J Eschmeier, K B Laursen, M M Neumann: Multipliers with natural local spectra on commutative Banach algebras, J Functional Analysis, to appear.

[7] D A Herrero: On the essential spectra of quasi-similar operators, Can. J Math. 40 (1988), 1436-1457. MR 90b:47006

[8] R Larsen: An introduction to the theory of multipliers, Springer-Verlag, New York 1971. MR 55:8695

[9] K B Laursen: Spectral subspaces and automatic continuity, Doctoral Dissertation, Copenhagen 1991.

[10] K B Laursen, M Mbekhta: Closed range multipliers and generalized inverses, Studia Math. 107 (2) (1993), 127-135. MR 94i:47052

[11] K B Laursen, V G Miller, M M Neumann: Local spectral properties of commutators, Proc. Edinburgh Math. Soc., 38 (1995), 313-329. MR 96f:47066

[12] K B Laursen, M M Neumann: Local spectral properties of multipliers on Banach algebras, Arch. Math. 58 (1992), 368-375. MR 93e:46058

[13] K B Laursen, M M Neumann: Asymptotic intertwining and spectral inclusions on Banach spaces, Czechoslovak Math. J 43 (118) (1993), 483-497. MR 94k:47007

[14] K B Laursen, M M Neumann: Local spectral theory and spectral inclusions, Glasgow Math. J 36 (1994), 331-343. MR 95k:47002

[15] K B Laursen, P Vrbova: Some remarks on the surjectivity spectrum of linear operators, Czechoslovak Math. J 39 (114) (1989), 730-739. MR 90m:47010

[16] M Mbekhta: Generalisations de la decomposition de Kato aux opérateurs paranormaux et spectraux, Glasgow Math. J 29 (1987), 159-175. MR 88i:47010

[17] M Mbekhta: Local spectrum and generalized spectrum , Proc. Amer. Math. Soc. 112 (1991), 457-463. MR 91i:47004

[18] T L Miller, V G Miller: Equality of essential spectra of quasisimilar operators with property $(\delta)$, Glasgow Math. J, to appear. CMP 96:08

[19] V G Miller, M M Neumann: Local spectral theory for multipliers and convolution operators, in Algebraic methods in operator theory, Birkhäuser, Boston, 1994.

[20] M M Neumann: Local spectral theory for operators on Banach space and applications to convolution operators on group algebras, in Seminar Notes in Functional Analysis and PDEs 1993/94, Department of Mathematics, Louisiana State University, Baton Rouge, LA, 1994.

[21] C Schmoeger: Ein Spektralabbildungssatz, Arch. Math. 55 (1990), 484-489. MR 92h:47007 
[22] C Schmoeger: On isolated points of the spectrum of a bounded linear operator, Proc. Amer.Math. Soc. 117 (1993), 715-719. MR 93d:47007

[23] T T West: A Riesz-Schauder theorem for semi-Fredholm operators, Proc. Royal Irish Acad. 87A (1987), 137-146. MR 89i:47020

[24] M Zafran: On the spectra of multipliers, Pac. J Math. 47 (1973), 609-626. MR 48:4653

Matematisk Institut, Københavns Universitet, Universitetsparken 5, DK-2100 KøbenHAVN $\varnothing$, DENMARK

E-mail address: laursen@math.ku.dk 